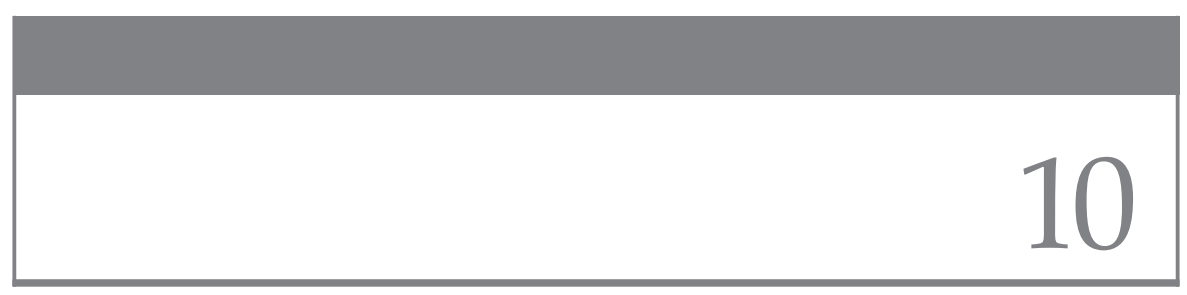

\title{
Using Technology to Advance Social Health
}

\author{
Hiroko H. Dodge
}

\section{INTRODUCTION}

Although the World Wide Web was developed less than 40 years ago, our societies, cultures, and way of life have been heavily impacted by this new information system. Many are now facing information overload through email, Facebook, Twitter, YouTube, and other social media. If we want to, we can surf the Internet and spend all day reading news and articles, posting comments, sharing pictures, and receiving comments from other people around the globe. In this chapter, social media is defined broadly by including any human interactions through the Internet such as email, group networking sites, and any commercial Internet network sites (e.g., dating sites), not limiting to big social media sites (e.g., YouTube, Facebook, Snapchat, Instagram, Twitter). According to the Pew Internet and American Life Project, 65\% of American adults used social media in 2005. This number has increased rapidly over the last 10 years and the trend includes seniors. In 2005, 2\% of seniors (age 65 and older) used social media compared with 35\% more recently (Perrin, 2015). Digital social networking is essentially limitless, but it requires an Internet connection, a device, and the ability to use the technology.

The questions to be addressed in this chapter include the following: (a) To what extent do older adults take advantage of the Internet and social media for their social interactions? (b) Is it possible to reduce the epidemic of social

This work is supported by R01AG051628, R01AG056102, R01AG033581,P30AG008017, and P30AG053760 from the National Institute of Health. I would like to thank Ms. Nora Mattek for her editing and proofreading. 
isolation through these technologies? The chapter is organized as follows: First, I will review some facts about how older adults are adopting new technologies and the barriers that prevent their adoption. Second, I will introduce new research areas which utilize modern technologies and have implications for combating the modern-day social isolation epidemic. Finally, I will discuss some cautions and research areas that need to be addressed before advocating digital socialization among older adults.

Before moving on, I would like to share some interesting discussion exchanges among members of the Gerontological Society of America (GSA) posted in its open forum a few months ago. A retired professor emeritus posted the following question in the GSA open forum.

I live in a CCRC (Continuing Care Retirement Community) where the community is very lively, stimulating, and we feel like a large family. Recently we are going through two periods of quarantine due to first, the flu, and secondly, a gastrointestinal infection. That has meant about three weeks without activities, van trips, and cautions against "congregating." My husband and I are healthy and can go out and about, but others are more limited. I would be interested in any research on what can be done to keep communication going during this kind of period. We have an email group with more than 60 members but there are many who are not on email. Does anyone have any experience with this?

In response to her post, a PhD student who has worked in care communities for 20 years responded by supporting the importance of keeping communities active when "quarantines" occur. She asked whether some technological system could be provided that would allow the opportunity to stay connected via computers (with a screen in each unit) and offering interactive games and conversation. The professor responded:

Thanks so much for your reply to my question. We are now nearing the end of the second week of quarantine for norovirus. At the end of January there was one week of quarantine for the flu. So, people are really missing the sense of community they enjoyed before. I think they were probably effective in limiting the infection. About 16 people (out of 300) had the norovirus but I felt it would be good to know specifically what healthy, uninfected people could reasonably do. We do have a not very good system called TouchTown which has a channel that most residents can access on their TV. At our suggestion, the exercise director did put some videos on there and people enjoyed doing exercises, knowing that others were doing them in their apartments. The activities director 
said she couldn't put on some of her videos, including Great Courses, because of technological limits to TouchTown. I think technology could be used more effectively. I was also wondering about more low-tech interactive activities, like art or book clubs. The email list was great for giving people the feel of a virtual community, but we do have a significant number of people who don't do email.

The previous comment was responded to by another GSA member as follows:

If residents have Internet access (or Internet-capable TVs), there's a site called TogetherTube that allows a group of people to watch the same YouTube video together at the same time. This might be good for exercise videos, documentaries, concerts, old movies, whatever, and would allow quarantined residents to share experiences with others. It has a built-in chat feature, too. Togethertube.com

These communications were posted under the thread title "Keeping socialization going during a quarantine in senior housing." I wanted to share this exchange because it elicits vivid images of lives in senior housing when such a quarantine strikes. Also, it provides important insights surrounding technology and seniors including that many do not use email and that the technology implemented in senior housing may not be optimal even though some devices are available. Even if useful technology is available (e.g., a built-in chat feature in YouTube), it may take time before the seniors find out about it. Therefore, it is likely to require initiations and training by the senior housing management teams before it will be translated into the daily lives of the seniors.

\section{TECHNOLOGY ADAPTABILITY AND BARRIERS: FACTS}

According to a 2014 Pew Research Center study, many older adults (those age 65 years and older) remain largely unattached from online and mobile life: $41 \%$ of seniors do not use the Internet at all, $53 \%$ do not have broadband access at home, and $23 \%$ do not use cell phones. Internet usage drops sharply as age increases. Among those age 80 years and older, only $21 \%$ have broadband at home. Looking at the existing data, Internet usage does not seem to have alleviated social isolation thus far. In fact, the data suggest that lack of Internet access could further widen the gap between older adults with abundant opportunities for social interaction and engagement and those with fewer opportunities. In the text that follows, I summarize potential factors thath may widen the gap in social interactions among seniors. 
First, broadband accessibility and Internet use depend highly on income and educational levels (Yu, Ellison, McCammon, \& Langa, 2016). Second, many seniors have physical conditions or health issues that make using these tools a challenge. Pew Research estimates that about $39 \%$ of older adults have physical conditions or health issues that prevent them from fully participating in many common daily activities and these seniors are significantly less likely than healthy seniors to go online ( $49 \%$ vs. $66 \%$ ), to have broadband at home ( $38 \%$ vs. $53 \%$ ), and to have a smartphone (13\% vs. $22 \%)$. Since those with physical limitations are more likely to be at risk of being home bound, this further contributes to the gap in social interaction opportunities. Third, there is a positive correlation between social media usage and socialization with others (i.e., those with high socialization tend to use social media sites more), according to the Pew Research. This correlation persists even after controlling for demographic factors such as age, income, or geographic area of residence. Fourth, older adults who do not use digital technologies are also less likely to believe that they are at a disadvantage by not using them: Only 35\% of older non-Internet users agree with the assessment that they are missing out on important information. On the other hand, over $79 \%$ of older adults who use the Internet agree with the statement that "people without Internet access are at a real disadvantage because of all the information they might be missing." And 94\% agree with the statement that "the Internet makes it much easier to find information today than in the past." In conclusion, there seems to be a spiral effect such that older adults with larger social networks may expand their network further using social media, while those who lack these social ties are less exposed to the opportunity of digital social media. As our society is more and more centered around Internet, those who do not have access to this information technology are being left behind; not only in terms of social network opportunities, but also a fundamental way of life such as getting essential information including community services and health resources. In the next section, I would like to introduce some new research efforts relevant to combating social isolation epidemics.

\section{POTENTIAL CONTRIBUTIONS OF TECHNOLOGY FOR COMBATING SOCIAL ISOLATION EPIDEMICS: NEW AREAS OF RESEARCH}

\section{Identifying Seniors With Social Isolation and Loneliness Objectively in Communities}

Those socially isolated rarely ask for help to alleviate their isolation. Furthermore, assessing loneliness in older adults is challenging owing to 
negative desirability biases associated with being lonely. Therefore, a priority is to develop more objective techniques to assess social isolation and loneliness in older adults so that preventive efforts may provide interventions before negative consequences accrue. Monitoring in-home daily activities continuously could identify subtle changes in behaviors that might indicate early onset of diseases but are likely to be missed if people are only assessed infrequently or at the time of emergency hospital visits. On the forefront of efforts to develop and apply more objective ecologically valid metrics of social isolation through home-based passive sensing and mobile health technologies is research led by the team at the Oregon Center for Aging and Technology (ORCATECH; www.orcatech.org), which was established over 10 years ago at Oregon Health \& Science University at Portland, OR (Kaye et al., 2011; Lyons et al., 2015). The center has been providing evidence that continuously monitored in-home activities such as walking speed (Dodge, Mattek, Austin, Hayes, \& Kaye, 2012; Kaye et al., 2012), sleep quality and duration (T. L. Hayes, Riley, Mattek, Pavel, \& Kaye, 2014; Seelye et al., 2015), driving patterns (Seelye et al., 2017), and computer usage (Kaye et al., 2014) could aid in identifying early signs of developing dementia, long before more obvious clinical symptoms emerge. This research has been focusing on detecting early dementia and related neurodegenerative disease, and extending the approach of capturing frequent objective activity measures to identifying not only structural aspects of the social network (infrequent social interactions), but also psychological outcomes of social isolation (loneliness or depression) (Petersen, Austin, Kaye, Pavel, \& Hayes, 2014; Petersen, Austin, Mattek, \& Kaye, 2015; Thielke et al., 2014). For example, by combining multiple metrics of in-home behaviors (gait speed, computer use, time out of home, phone use), algorithms have been identified that predict loneliness (Austin et al., 2016). Monitoring daily behavioral patterns and their changes over time at home have a major advantage compared with the traditional infrequent and brief assessment conducted at clinic visits, since the signals detected allow one to examine intraindividual changes as opposed to only applying normative group data (e.g., group means and median, etc.) to identify individual specific "deviations" or signals. The use of intraindividual changes over time enhances signal-to-noise ratios, providing more powerful metrics for the identification of the early onset of diseases (Dodge, Zhu, Mattek, Austin, et al., 2015). Findings suggest that involvement in this unobtrusive in-home monitoring approach is well accepted, and older adults are, in general, willing to share information from monitoring systems with family members and clinicians (Boise et al., 2013). Recently, the monitoring platform has been further refined through an National Institutes of Healh-Veterans Administration (NIH-VA) supported initiative to make it readily deployable 
to a larger number of households across the larger research community and to include diverse populations (low income, rural, ethnic minorities [www. carthome.org]). Wearable mobile technology (mHealth) could also potentially contribute to the early identification of social isolation in the future, although to our knowledge, none of the current interventions using mHealth are specifically aimed to alleviate social isolation or loneliness (Rathbone \& Prescott, 2018).

\section{Randomized Controlled Trials (RCTs) Targeting Socially Isolated Seniors to Enhance Their Social Interactions}

Can we enhance social interactions through Internet use and improve the psychological well-being and cognitive function of socially isolated older adults? Our team, I-CONECT (Internet-Based Conversational Engagement Clinical Trials, www.i-conect.org), is conducting a series of RCTs that aims to examine whether digital social interactions, specifically video chat with interviewers, can improve cognitive functions and psychological well-being among socially isolated seniors. In our previous proof of concept study, which was completed in 2014 (Dodge, Zhu, Mattek, Bowman, et al., 2015) (Clinicaltrials.gov registration number NCT01571427), the experimental group received daily (five times per week) 30-minute conversations with interviewers while the control group received only once per week check-in calls. Despite a short duration of 6 weeks, we found improved cognitive functions in the domains of language-based executive function and semantic memory among the experimental group compared with the control group (Dodge, Zhu, Mattek, Bowman, et al., 2015). This pilot study was not limited to those with social isolation. Building on these promising results and foundation work, our team is currently conducting a follow-up study, specifically targeting social isolated seniors age 75 and older (Clinicaltrials.gov registration number NCT02871921). The participants are recruited mainly from the local Meals on Wheels programs. Although the primary outcomes of this RCT are cognitive functions (i.e., preintervention and postintervention changes in domain-specific cognitive functions), we are also examining whether change in psychological well-being, such as mood and loneliness, mediates or moderates the effect of social interaction on cognition and, if so, to what extent. In this project, social isolation is operationally defined using Lubben's Social Isolation Score, 6 items version (LSNS-6; Lubben et al., 2006) of 12 or lower. The study has a duration of 12 months of intervention with two "doses" of conversational engagement or "chatting" (4 days per week for 6 months and 2 days per week for 6 months). These projects are the 
first series of prevention trials examining the effects of social interactions on cognitive reserve, targeting the socially isolated seniors who are less likely to volunteer for research despite their high risk of cognitive impairment. Besides recruiting from an underserved population, unique features of this project include the use of preintervention and postintervention changes in brain structures and integrities assessed by volumetric and resting state functional MRI to clarify the underlying mechanisms of efficacies. Exploratory outcomes include objective assessment of instrumental activities of daily living using an electronic pillbox that collects time-stamped data when the box is opened (i.e., to monitor medication adherence) and changes in speech characteristics over time. These exploratory outcomes are introduced based on the promising results from the previous trial. For example, using the transcribed conversations between interviewers and participants recorded during the video chats, the team found that speech characteristics could distinguish early mild cognitive impairment from those with normal cognition (Asgari, Kaye, \& Dodge, 2017; Dodge et al., 2015). Data on personality is also being collected as a potential effect modifier. The results of the RCT will be available in the year 2023. Lancet Commissions (Livingston et al., 2017) recently estimated that $2 \%$ of people with dementia occurs because of social isolation among seniors. A comparative figure of population attributable risk (PAR) of diabetes was estimated to be only $1 \%$. That is, the impact of social isolation on dementia is higher than that of diabetes among seniors. The results of this RCT may answer questions initiated by epidemiological studies such as, "What are the effects of social interactions on brain integrity, psychological well-being, and loneliness?" and "What are the mediation effects of improved psychological well-being on the association between cognitive functions and social interactions?" If this RCT confirms promising results, we plan to deliver the approach identified in this trial to a larger community, scalable to a national level, for example, by introducing video chat with seniors as a part of college gerontology program curriculums.

\section{Development of User-Friendly Multifunctional Devices}

In addition to identifying social isolation at the earliest stage and providing social interactions through the Internet (e.g., video chats) as discussed earlier, another area of technology-intensive research is the development of userfriendly devices that can be deployed to a large number of seniors cost effectively. A significant majority of older adults need assistance when using new digital devices. According to the Pew Research study, just 18\% would feel comfortable learning to use a new technology device such as a smartphone 
or tablet on their own, while 77\% indicate they would need someone to help walk them through the process. Among seniors who go online but do not currently use social networking sites such as Facebook or Twitter, 56\% would need assistance if they wanted to use these sites to connect with friends or family members. Recently Czaja and colleagues (Czaja, Boot, Charness, Rogers, \& Sharit, 2017) reported their trial results evaluating the impact of a specially designed computer system, PRISM (Personal Reminder Information and Social Management), for social isolation, loneliness, and well-being. The PRISM device contains various easy-to-use senior-focused features including Internet access with vetted links, an annotated resource guide, a dynamic classroom feature, a calendar, a photo feature, email access, games, and online help. In the 12-month trial, the experimental group was compared to a control condition that included a binder containing content that paralleled the PRISM system in a nonelectronic form (e.g., paper resource guides, paper calendar). They found that at 6 months, those who received PRISM reported significantly decreased loneliness and increased perceived social support and well-being as compared with those who received the binder. Although the difference between the experimental and the control groups was not significant at the 12-month assessment, this study showed that userfriendly devices like PRISM are well accepted among seniors. The study also provided evidence that by making this type of user-friendly device available to larger and more diverse populations through cost reductions via a public-private partnership, we might be able to reduce social isolation among seniors and enhance their psychological well-being.

\section{The Efforts to Narrow the Digital Gap: National Efforts}

There have been some national movements to narrow the digital gap. For example, ConnectHomeUSA (formerly ConnectHome Nation) is an organization that started in 2012 to bridge the digital divide for Housing and Urban Development (HUD)-assisted housing residents in the United States in collaboration with the national nonprofit EveryoneOn. It provides free or lowcost broadband access, devices, and digital literacy training by creating a platform for community leaders, local governments, nonprofit organizations, and private industry to join together and produce locally tailored solutions for narrowing the digital divide. Originally, the aim of ConnectHomeUSA was to provide educational tools for school-age children in HUD-assisted housing, but the platform has been extended to assist seniors. For example, in 2017, the Jewish Healthcare Foundation in Pittsburgh, PA, launched Virtual Senior Academy (virtualsenioracademy.org), which is a free interactive 
platform that offers virtual classes through video conferences. It brings seniors together without leaving their homes. They recruit Virtual Senior Academy facilitators of all ages and lead classes. The project is largely run by volunteers. Although the senior participation rate is increasing rapidly, those without Internet or a computer have no way to join. The foundation is currently working with ConnectHomeUSA to alleviate this barrier to access by bringing Internet connections and providing devices to senior high rises in the area. More information is available at the ConnetHomeUSA website.

\section{DIGITAL SOCIAL NETWORK USAGE AND PSYCHOLOGICAL WELL-BEING: SOME CAUTIONS}

So far, my discussion was centered on enhancing Internet usage among seniors to alleviate social isolations. In this section, I would like to provide some cautions for doing so. Recent studies on Internet social network site use and psychological well-being among seniors provided some positive results. For example, using the 2012 Health and Retirement Study (HRS), Yu, McCammon, Ellison, and Langa (2015) showed that among those age 50 and older, Internet social network site use is positively associated with social well-being outcomes, including perceived support from friends and feelings of connectedness, and perceived support from children. Other cross-sectional studies also showed that the frequency of going online (Cotten, Anderson, \& McCullough, 2013) was associated with decreased loneliness scores. One Australian study reported that greater use of the Internet as a communication tool was associated with a lower level of social loneliness, but greater use of the Internet to find new people was associated with a higher level of emotional loneliness (Sum, Mathews, Hughes, \& Campbell, 2008). These studies tend to have a smaller sample size with a wide age range (e.g., including lower age bound of 50) or are limited to cross-sectional analyses and to those who are already Internet users. Studies with a more inclusive sample, assessments of long-term effects, and age-stratified analyses (e.g., younger old vs. oldest old) are warranted. Among studies with younger participants, recent meta-analyses showed that social network usage does not necessarily lead to improved psychological well-being and psychological connectedness (Verduyn, Ybarra, Résibois, Jonides, \& Kross, 2017). The study found that especially passive usage (e.g., monitoring of other people's posts without engaging in direct exchanges with others such as scrolling through news feeds or looking at other users' profiles, pictures, and status updates) of social network sites has a negative impact on subjective well-being, possibly because it provokes social comparisons and envy, while active usage of social 
network sites predicts subjective well-being by creating social capital and stimulating feelings of social connectedness. Older adults use social media less actively than young adults (i.e., they are likely to be more passive users) (M. Hayes, van Stolk-Cooke, \& Muench, 2015). Few investigators have examined granular usage patterns and psychological well-being among seniors. More research is needed before prescribing Internet and social media use among seniors.

Another area that should not be ignored is potential benefits of nondigital in-person interactions. It is well documented that nonsexual physical contact such as hugging and handholding has stress-buffering effects and may improve perceived feelings of social support. For example, Cohen, JanickiDeverts, Turner, and Doyle (2015) examined whether global perceptions of social support and the actual receipt of physical touch or hugging during daily life attenuated the association of an interpersonal stressor (social conflict) with subsequent risk for infection, cold signs, and clinical disease in response to an experimentally administered cold virus. The authors found people who receive more hugs are more protected from infection and illness-related symptoms. A number of laboratory studies also show that touch from a trusted individual buffers the effects of stress. The well-known handholding study by Coan showed that married women were subjected to the threat of electric shock to a lesser extent while holding their husband's hand, in comparison with holding a stranger's hand. Furthermore, the extent of the threat reduction was associated with the quality of the relationship with their husband (Coan, Schaefer, \& Davidson, 2006). Encouraging seniors to stay connected without leaving their house through enhanced digital social engagement may be beneficial to their well-being. However, relying on this platform heavily could leave out some important aspects of social interactions that exist only in traditional humanto-human encounters. Advocating digital media usage without confirming its possible negative effects or "side effects" has risks.

Finally, cybercrime has increased dramatically over the years. Seniors, especially those with impaired cognitive abilities or those socially isolated without anyone to consult with, are particularly vulnerable to becoming a victim of identity theft or Internet fraud by opening spam mail, catching computer viruses, or being lured by illegitimate advertisement sites. Older adults have a diverse range of cognitive ability as well as large variation in their computer proficiency. Identifying specific pathways through which seniors are more likely to be victimized and developing effective prevention against cybercrimes is urgently needed. Enhancement of social media access has to be accompanied by educational opportunities against cybercrimes. 


\section{SUMMARY}

In this chapter, I summarized the current state of Internet and social media usage among seniors and discussed the digital divide among older adults which may further expand the gap in social interaction opportunities. Then three new research initiatives were discussed that use modern technology intensively and have implications for social isolation epidemics. Finally, some cautions were raised in advocating social media use among seniors. Recent technological advancement is unprecedented. I am optimistic that these technologies can alleviate social isolation epidemics as long as we do not blindly facilitate the use of these technologies among seniors.

\section{REFERENCES}

Rathbone, A. L., \& Prescott, J. (2018). The use of mobile apps and SMS messaging as physical and mental health interventions: Systematic review. Journal of Medical Internet Research, 19(8), e295. doi:10.2196/jmir.7740

Asgari, M., Kaye, J., \& Dodge, H. (2017). Predicting mild cognitive impairment from spontaneous spoken utterances. Alzheimers Dement (N Y), 3(2), 219-228. doi:10.1016/ j.trci.2017.01.006

Austin, J., Dodge, H. H., Riley, T., Jacobs, P. G., Thielke, S., \& Kaye, J. (2016). A smart-home system to unobtrusively and continuously assess loneliness in older adults. IEEE Journal of Translational Engineering in Health and Medicine, 4, 2800311. doi:10.1109/ JTEHM.2016.2579638

Boise, L., Wild, K., Mattek, N., Ruhl, M., Dodge, H. H., \& Kaye, J. (2013). Willingness of older adults to share data and privacy concerns after exposure to unobtrusive in-home monitoring. Gerontechnology, 11(3), 428-435. doi:10.4017/gt.2013.11.3.001.00

Coan, J. A., Schaefer, H. S., \& Davidson, R. J. (2006). Lending a hand: Social regulation of the neural response to threat. Psychological Science, 17(12), 1032-1039. doi:10.1111/j.1467-9280.2006.01832.x

Cohen, S., Janicki-Deverts, D., Turner, R. B., \& Doyle, W. J. (2015). Does hugging provide stress-buffering social support? A study of susceptibility to upper respiratory infection and illness. Psychological Science, 26(2), 135-147. doi:10.1177/ 0956797614559284

Cotten, S. R., Anderson, W. A., \& McCullough, B. M. (2013). Impact of Internet use on loneliness and contact with others among older adults: Cross-sectional analysis. Journal of Medical Internet Research, 15(2), e39. doi:10.2196/jmir.2306

Czaja, S. J., Boot, W. R., Charness, N., Rogers, W. A., \& Sharit, J. (2017). Improving social support for older adults through technology: Findings from the PRISM randomized controlled trial. Gerontologist, 58(3), 467-477. doi:10.1093/geront/gnw249

Dodge, H. H., Mattek, N. C., Austin, D., Hayes, T. L., \& Kaye, J. A. (2012). In-home walking speeds and variability trajectories associated with mild cognitive impairment. Neurology, 78(24), 1946-1952. doi:10.1212/WNL.0b013e318259e1de

Dodge, H. H., Mattek, N., Gregor, M., Bowman, M., Seelye, A., Ybarra, O., . . Kaye, J. A. (2015). Social Markers of mild cognitive impairment: Proportion of word counts in free conversational speech. Current Alzheimer Research, 12(6), 513-519. doi:10.2174/15 67205012666150530201917 
Dodge, H. H., Zhu, J., Mattek, N. C., Austin, D., Kornfeld, J., \& Kaye, J. A. (2015). Use of high-frequency in-home monitoring data may reduce sample sizes needed in clinical trials. PLoS One, 10(9), e0138095. doi:10.1371/journal.pone.0138095

Dodge, H. H., Zhu, J., Mattek, N., Bowman, M., Ybarra, O., Wild, K., \& Kaye, J. A. (2015). Web-enabled conversational interactions as a means to improve cognitive functions: Results of a 6-week randomized controlled trial. Alzheimers Dement (N Y), 1(1), 1-12. doi:10.1016/j.trci.2015.01.001

Hayes, M., van Stolk-Cooke, K., \& Muench, F. (2015). Understanding Facebook use and the psychological affects of use across generations. Computers in Human Behavior, 49, 507-511. doi.org/10.1016/j.chb.2015.03.040

Hayes, T. L., Riley, T., Mattek, N., Pavel, M., \& Kaye, J. A. (2014). Sleep habits in mild cognitive impairment. Alzheimer Disease and Associated Disorders, 28(2), 145-150. doi:10.1097/WAD.0000000000000010

Kaye, J., Mattek, N., Dodge, H., Buracchio, T., Austin, D., Hagler, S., . . Hayes, T. (2012). One walk a year to 1000 within a year: Continuous in-home unobtrusive gait assessment of older adults. Gait Posture, 35(2), 197-202. doi:10.1016/j.gaitpost.2011.09.006

Kaye, J., Mattek, N., Dodge, H. H., Campbell, I., Hayes, T., Austin, D., . . Pavel, M. (2014). Unobtrusive measurement of daily computer use to detect mild cognitive impairment. Alzheimers \& Dementia, 10(1), 10-17. doi:10.1016/j.jalz.2013.01.011

Kaye, J. A., Maxwell, S. A., Mattek, N., Hayes, T. L., Dodge, H., Pavel, M., . . Z Zitzelberger, T. A. (2011). Intelligent systems for assessing aging changes: Home-based, unobtrusive, and continuous assessment of aging. Journals of Gerontology. Series B, Psychological Sciences and Social Sciences, 66 Suppl 1, i180-i190. doi:10.1093/geronb/gbq095

Livingston, G., Sommerlad, A., Orgeta, V., Costafreda, S. G., Huntley, J., Ames, D., . . . Mukadam, N. (2017). Dementia prevention, intervention, and care. Lancet, 390(10113), 2673-2734. doi:10.1016/S0140-6736(17)31363-6

Lubben, J., Blozik, E., Gillmann, G., Iliffe, S., von Renteln Kruse, W., Beck, J. C., \& Stuck, A. E. (2006). Performance of an abbreviated version of the Lubben Social Network Scale among three European community-dwelling older adult populations. Gerontologist, 46(4), 503-513. doi:10.1093/geront/46.4.503

Lyons, B. E., Austin, D., Seelye, A., Petersen, J., Yeargers, J., Riley, T., . . Kaye, J. A. (2015). Pervasive computing technologies to continuously assess Alzheimer's disease progression and intervention efficacy. Frontiers in Aging Neuroscience, 7, 102. doi:10.3389/ fnagi.2015.00102

Perrin, A. (2015). Social Nnetworking Uusage: 2005-2015. Retrieved from http://www. pewinternet.org/2015/10/08/social-networking-usage-2005-2015

Petersen, J., Austin, D., Kaye, J. A., Pavel, M., \& Hayes, T. L. (2014). Unobtrusive in-home detection of time spent out-of-home with applications to loneliness and physical activity. IEEE Journal of Biomedical and Health Informatics, 18(5), 1590-1596. doi:10.1109/ JBHI.2013.2294276

Petersen, J., Austin, D., Mattek, N., \& Kaye, J. (2015). Time out-of-home and cognitive, physical, and emotional wellbeing of older adults: A longitudinal mixed effects model. PLoS One, 10(10), e0139643. doi:10.1371/journal.pone.0139643

Seelye, A., Mattek, N., Howieson, D., Riley, T., Wild, K., \& Kaye, J. (2015). The impact of sleep on neuropsychological performance in cognitively intact older adults using a novel in-home sensor-based sleep assessment approach. Journal of Clinical Neuropsychology, 29(1), 53-66. doi:10.1080/13854046.2015.1005139 
Seelye, A., Mattek, N., Sharma, N., Witter, P., Brenner, A., Wild, K., . . Kaye, J. (2017). Passive assessment of routine driving with unobtrusive sensors: A new approach for identifying and monitoring functional level in normal aging and mild cognitive impairment. Journal of Alzheimers Disease, 59(4), 1427-1437. doi:10.3233/JAD-170116

Sum, S., Mathews, R. M., Hughes, I., \& Campbell, A. (2008). Internet use and loneliness in older adults. Cyberpsychology and Behavior, 11(2), 208-211. doi:10.1089/cpb.2007.0010

Thielke, S. M., Mattek, N. C., Hayes, T. L., Dodge, H. H., Quinones, A. R., Austin, D., ... Kaye, J. A. (2014). Associations between observed in-home behaviors and self-reported low mood in community-dwelling older adults. Journal of the American Geriatrics Society, 62(4), 685-689. doi:10.1111/jgs.12744

Verduyn, P., Ybarra, O., Résibois, M., Jonides, J., \& Kross, E. (2017). Do social network sites enhance or undermine subjective well-being? A critical review. Social Issues and Policy Review, 11(1), 274-302. doi:10.1111/sipr.12033

Yu, R. P., Ellison, N. B., McCammon, R. J., \& Langa, K. M. (2016). Mapping the two levels of digital divide: Internet access and social network site adoption among older adults in the USA. Information, Communication \& Society, 19(10), 1445-1464. doi:10.1080/136 9118X.2015.1109695

Yu, R. P., McCammon, R., Ellison, N., \& Langa, K. (2015). The relationships that matter: Social network site use and social wellbeing among older adults in the United States of America. Ageing and Society, 36(9), 1826-1852. doi:10.1017/S0144686X15000677 
